\title{
HÉROÏSME ET EXOTISME. LE MILITAIRE À L'ÉPREUVE DES LOINTAINS (1820-1914)
}

Hervé Mazurel

Armand Colin | « Romantisme »

2013/3 n 161 | pages 35 à 44

ISSN 0048-8593

ISBN 9782200928841

Article disponible en ligne à l'adresse :

http://www.cairn.info/revue-romantisme-2013-3-page-35.htm

\section{Pour citer cet article :}

Hervé Mazurel, « Héroïsme et exotisme. Le militaire à l'épreuve des lointains (1820-1914) », Romantisme 2013/3 (n¹61), p. 35-44.

DOI 10.3917/rom.161.0035

Distribution électronique Cairn.info pour Armand Colin.

(C) Armand Colin. Tous droits réservés pour tous pays.

La reproduction ou représentation de cet article, notamment par photocopie, n'est autorisée que dans les limites des conditions générales d'utilisation du site ou, le cas échéant, des conditions générales de la licence souscrite par votre établissement. Toute autre reproduction ou représentation, en tout ou partie, sous quelque forme et de quelque manière que ce soit, est interdite sauf accord préalable et écrit de l'éditeur, en dehors des cas prévus par la législation en vigueur en France. Il est précisé que son stockage dans une base de données est également interdit. 


\section{Héroïsme et exotisme. Le militaire à l'épreuve des lointains (1820-1914)}

Il est d'ordinaire coutume de présenter le XIX ${ }^{\mathrm{e}}$ siècle comme un siècle peu guerrier. Et, à en juger par ses bornes, il l'est peu en effet. Il clôt d'un côté une longue séquence guerrière d'un quart de siècle, issue des secousses de la Révolution, tandis qu'il sombre, de l'autre, dans le grand cataclysme de 14-18. Dans l'intervalle, le continent européen n'a connu que de rares conflits et, hormis la guerre de Crimée, des affrontements relativement circonscrits tant dans la durée que par le nombre des belligérants. Si le constat paraît juste a priori, il demeure toutefois prisonnier du souvenir-écran qu'imposa le traumatisme de la Grande Guerre, qui a longtemps interdit d'évaluer à sa juste valeur la place du fait guerrier au cours du long XIX ${ }^{\mathrm{e}}$ siècle. La taille et le souvenir d'une guerre franco-prussienne prémonitoire, donc restée en lumière, semblent avoir laissé dans l'ombre d'innombrables conflits, petits et moyens et effacé des mémoires la forte belliquosité du temps. Il serait en cela plus juste de dire que les grandes puissances n'ont pas tant établi la paix en 1815 qu'elles n'ont déplacé la guerre au-delà des mers ${ }^{1}$. Qui se penche sur la chronologie fine du siècle et observe l'Europe du « dehors » et non du « dedans " ne peut en effet que constater l'étonnante fréquence des conflits dans lesquels des corps de soldats européens se sont engagés au loin, tout particulièrement en Asie et en Afrique. Souvent jugés mineurs et périphériques, puisque mesurés à l'aune de cette immense conflagration continentale, il importe de restituer aujourd'hui à ces conflits éloignés toute leur dignité historiographique.

Le cas français est, avec l'anglais, le plus intéressant car il regorge d'expériences militaires lointaines et exotiques, le plus souvent très loin de l'Europe, à ses marges parfois. Songeons, bien sûr, aux 37000 soldats qui s'embarquèrent à Toulon, le 16 mai 1830, à la conquête d'Alger, aux 32000 Français qui firent l'expérience des chaleurs mexicaines entre 1862 et 1867, aux 35000 hommes, encore, partis conquérir le Tonkin, la Chine en ligne de mire. Mais rappelons-nous également les milliers de vétérans napoléoniens moins connus, errant de part le monde dans l'Internationale libérale ou comme mercenaires au cours des années 1820, les 15000 soldats de la campagne de Morée de 1828, les 7000 français oubliés de l'expédition de Chine en 1860, les 15000 hommes envoyés pour la prise de Madagascar en 1895. Et ce serait là encore omettre les dizaines de milliers de soldats qui s'échinèrent à

1. David Bell, The First Total War : Napoleon's Europe and the Birth of Warfare as We Know It, New York, Houghton Mifflin, 2007, p. 309. 
soumettre l'Afrique occidentale et équatoriale au cours des années 1880-1890, ceux des fameuses colonnes méharistes mis à l'épreuve du Sahara, ceux, encore, qui tinrent tête à Pékin en 1900 à la révolte des Boxers, ceux, enfin, près de 60 000, envoyés au Maroc à la veille du Premier conflit mondial.

Par là, la guerre au loin, qu'elle ait été vécue aux creux des déserts ou des forêts vierges, dans des montagnes reculées ou au milieu de villes inconnues et parfois surpeuplées, fut en France une expérience masculine bien plus massive qu'on ne l'imagine souvent. Pour sûr, elle imprégna en profondeur l'imaginaire social ${ }^{2}$, bouleversant, dans cette abondance d'images-forces, les représentations du militaire au XIX ${ }^{\mathrm{e}}$ siècle. Avec l'avènement de la culture de masse, dès les années $1860^{3}$, ces guerres lointaines ont constitué pour les Français un décor de plus en plus prégnant au fil du siècle. À observer par exemple, en sa dernière décennie, les unes du Petit journal ou de l'Illustration, sinon les couvertures illustrées de la littérature enfantine ou des romans populaires, l'on voit sans cesse se rejouer les mêmes scènes et intrigues : celles de combats exotiques, presque toujours victorieux, de troupes européennes contre des guerriers autochtones, d'Afrique, d'Asie, d'Amérique ou des mers du Sud. Bien que les motifs, les lieux et les acteurs different, ces images ne cessent au fond de conjuguer avec force hérö̈sme et exotisme et de renforcer leurs liens au sein des systèmes de valeurs dominants. Que ces guerres éloignées aient été un lieu de désir collectif - une matière à fantasmes - à l'heure où l'anxiété des pathologies urbaines et industrielles suscitait une soif d'évasion, et qu'elles aient été le creuset d'une image nouvelle et valorisée du militaire, voilà qui dessine l'objet des lignes qui suivent.

\section{LE MONDE COMME CHAMP DE BATAILLE}

Il convient, pour comprendre la puissance croissante de ce couple, de mieux saisir la manière dont le monde s'est constitué dès les années 1820 en nouveau champ de bataille des guerriers venus d'Europe. Et il faut pour cela relier avec davantage de force que l'historiographie courante les sorties de guerre napoléoniennes et le déploiement des conquêtes coloniales ${ }^{4}$. Car il est un événement central, consubstantiel à Waterloo, qui éclaire le déploiement de ce nomadisme militaire à l'échelle du siècle : l'éclipse des champs de bataille en Europe après 1815. Et, avec elle, l'anéantissement des rêves de gloire, des possibilités d'héroïsme.

2. On reprendra ici à Dominique Kalifa sa précieuse définition de l'imaginaire social « comme système cohérent, dynamique, de représentations du monde social, une sorte de répertoire des figures et des identités collectives dont se dote chaque société à des moments donnés de son histoire ». Les imaginaires sociaux décrivent encore « la façon dont les sociétés perçoivent leurs composants - groupes, classes, catégories -, hiérarchisent leurs divisions, élaborent leur avenir. Ils produisent et instituent le social plus qu'ils ne le reflètent. Mais ils ont besoin pour cela de s'incarner dans des intrigues, de raconter des histoires, de les donner à lire ou à voir ». Dominique Kalifa, Les Bas-Fonds. Histoire d'un imaginaire, Paris, Seuil, 2013, p. 20-21.

3. Sur cette naissance plus précoce qu'annoncée d'ordinaire : Dominique Kalifa, La Culture de masse, 1860-1930, Paris, La Découverte, 2001.

4. Que cherchait Charles X en Algérie en 1830 sinon le ré-ancrage de son régime dans une victoire militaire flamboyante à même de renouer, dans l'opinion, avec les gloires de l'Empire ? 
Ce brutal ralentissement du pouls du temps provoqué par l'effacement de l'épopée napoléonienne toucha en priorité deux catégories d'hommes, demeurés peu sensibles au reflux ambiant de l'idée militaire ${ }^{5}$. En premier lieu, la masse des vétérans, des "vieux " soldats démobilisés, ceux qui considéraient la guerre comme leur véritable métier et se montraient incapables de revenir à une vie civile sans éclat. De là, dans leurs rangs, un fort attrait pour l'émigration guerrière ${ }^{6}$. Certains, forts de leurs idéaux libéraux ou républicains, épousèrent d'emblée le circuit révolutionnaire mondial, s'en allant notamment combattre auprès de Bolivar et de San Martin. Comme des centaines d'officiers français, ce fut le cas d'un Georges Beauchef. Volontaire en 1805, l'homme avait combattu à Austerlitz, Iéna et Friedland. Fait officier au cours des Cent-Jours, il honnissait les Bourbons et passa dès la chute de l'Empire en Argentine, y faisant tôt étalage de ses talents militaires. Son autorité naturelle et ses qualités manœuvrières le distinguèrent surtout lors des campagnes pour l'indépendance du Chili entre 1817 et $1825^{7}$. D'autres, aux logiques mercenaires plus affichées, en vinrent à offrir leur savoir-faire au plus offrant, et ce jusqu'en Inde comme JeanFrançois Allard et Jean-Baptiste Ventura formant en 1822 les troupes du Pendjab ${ }^{8}$, mais le plus souvent dans l'Orient turc, égyptien ou perse. Tandis qu'après Waterloo, l'aîné des frères Bacheville partit servir le fils du Shah, son cadet, ex-capitaine de la Vieille Garde, très surveillé des Bourbons, tenta sa chance en Albanie auprès du célèbre tyran Ali Pacha de Ioannina'. Mais, comme le montre l'itinéraire d'un Maurice Persat, qui combattit auprès des insurgés italiens, espagnols, grecs et latinoaméricains, mais qui se fit également flibustier dans les Caraïbes et aux États-Unis, avant de réintégrer l'armée française pour les campagnes de Morée et d'Algérie, les frontières entre volontariat et mercenariat n'étaient jamais totalement étanches ${ }^{10}$. Tout pousse par ailleurs, on le voit, à penser ensemble l'histoire des révolutions libérales et démocratiques, celle des guerres d'indépendance nationale et celle des conquêtes coloniales.

Le second groupe puisait dans la génération romantique, élevée dans le bruit des combats et la gloire du sabre, chez ceux dont Vigny disait, avant Musset, qu'ils étaient comme perdus « entre le rêve et l'écho des batailles ${ }^{11}$ ". Soit toute une jeunesse romantique qui s'était préparée à la guerre et qui, surprise par la paix, se mit en quête d'ailleurs guerriers où passer l'épreuve du feu et égaler l'héroïsme paternel ${ }^{12}$. Arrimée

5. Raoul Girardet, La Société militaire de 1815 à nos jours, Paris, Perrin, 1998, p. 13-22. Voir aussi Geoffroy Best, War and Society in Revolutionary Europe, 1770-1870, Londres, McGill-Queens University Press, 1998.

6. Jean Vidalenc, Le Demi-solde. Étude d'une catégorie sociale, Paris, Librairie Marcel Rivière, 1955 ; Nathalie Petiteau, Lendemains d'Empire. Les soldats de Napoléon dans la France du XIXe siècle, Paris, La Boutique de l'histoire, 2003. 2001.

7. Georges Beauchef, Mémoires pour servir à l'indépendance du Chili (1822), Paris, La Vouivre,

8. Walter Bruyère-Ostells, Histoire des mercenaires, Paris, Taillandier, 2011, p. 42-43.

9. Voyages des frères Bacheville, capitaines de l'ex-garde, en Europe et en Asie, Paris, Imprimerie de David, 1822

10. Maurice Persat, Mémoires, 1806-1844, Plon-Nourrit, Paris, 1910.

11. Alfred de Vigny, Servitudes et grandeur de la vie militaire [1835], Paris, LGF, 1965, p. 22.

12. Voir notamment Déborah Guterman, « Le désir et l'entrave. L'impuissance dans la construction de l'identité masculine romantique : première moitié du XIX ${ }^{\mathrm{e}}$ siècle », dans Régis Révenin (dir.), Hommes 
au mal du siècle, cette soif de combat naissait d'abord du besoin d'une initiation virile qui leur était refusée depuis 1815. Après vingt-cinq années de guerre, être homme supposait un baptême du feu. Or, c'est pour satisfaire à ce rite de passage qu'un François-Blaise Schack s'était porté volontaire pour la Grèce ${ }^{13}$ ou qu'un Jules Lefevre-Deumier se rendit en Pologne. "Las de la vie de garnison » et de " plusieurs années d'oisiveté sans combattre ${ }^{14}$ ", le jeune officier Achille de Saint-Arnaud, le futur maréchal, tout juste 22 ans, démissionna ainsi en 1821 pour se battre en Grèce. Car il ne s'agissait pas simplement d'être soldat, mais d'être un guerrier, avec cette expérience du feu qui, seule, confere la valeur. Aux jeunes militaires de carrière, il était intolérable de moisir dans des garnisons reculées sans avoir l'occasion de se couvrir de gloire. D'où l'enthousiasme retrouvé du même Saint-Arnaud, dix ans plus tard, à son départ pour l'Algérie ${ }^{15}$. Ainsi est-ce avant tout à la croisée de ces nostalgies et de ces impatiences que s'enracina chez cette jeunesse frustrée d'action la décision de gagner ces horizons lointains.

Tandis que les vieux soldats ne pouvaient sortir du cercle de la guerre, les plus jeunes, eux, ne semblaient rêver que d'une chose : y entrer ${ }^{16}$. C'est que le modèle militaro-viril, issu des profonds bouleversements des guerres de la Révolution et de l'Empire, pesait de tout son poids sur les uns et les autres ${ }^{17}$. Il avait installé chez les premiers la peur de l'efféminement et d'une soudaine dévirilisation, tandis qu'il avait creusé chez les seconds l'angoisse d'être à jamais exclu, faute de guerres, du monde des hommes. D'où l'importance de s'en aller quêter un champ de bataille en ces théâtres lointains, quitte à devoir pour cela errer jusqu'aux confins du monde ${ }^{18}$.

\section{LES NOUVELLES NOCES DE LA GUERRE ET DU VOYAGE}

Que la guerre ait été à ce point objet de désir ? Cette idée nous est presque devenue étrangère tant les atrocités des deux guerres mondiales ont profondément déromantisé l'activité guerrière ${ }^{19}$. Mais, en ce temps où l'enthousiasme belliqueux aimait à s'épanouir publiquement, la guerre était moins jugée cruelle et sordide que belle et glorieuse, parce qu'arrimée à cette prégnante culture romantique de l'héroïsme

et masculinités de 1789 à nos jours. Contributions à l'histoire du genre et de la sexualité en France, Paris, Autrement, 2007, p. 55-74 ; Margaret Waller, The Male Malady : Fictions of Impotence in French Romantic Novels, New Brunswick, Rutgers University Press, 1993.

13. François-Blaise Schack, Campagne d'un jeune Français en Grèce, Paris, Firmin Didot, 1827.

14. François Maspero, L'Honneur de Saint-Arnaud, Paris, Plon, 1993, p. 35.

15. Lettres du maréchal de Saint-Arnaud, 1832-1854, publiées par Ad. Leroy de Saint-Arnaud et précédées d'une notice de Sainte-Beuve, Paris, M. Levy frères, 1858.

16. Jean-Paul Bertaud, «La virilité militaire » dans Alain Corbin, Jean-Jacques Courtine, Georges Vigarello (dir.), Histoire de la virilité, Paris, Seuil, 2011, t. II, p. 157-202.

17. George L. Mosse, The Image of the Man. The Creation of Modern Masculinity, New York, Oxford University Press, 1996 ; André Rauch, Crise de l'identité masculine, 1789-1914, Paris, Hachette, 2001 ; Anne-Marie Sohn, "Sois un homme ». La Construction de la masculinité au XIXe siècle, Paris, Seuil, 2009.

18. Sur ces circulations massives de soldats européens, mêlant vieux soldats et jeunes romantiques, mercenaires et volontaires, voir Walter Bruyère-Ostells, La Grande Armée de la liberté, Paris, Taillandier, 2009, et Histoires de mercenaires, Paris, Taillandier, 2011.

19. Stéphane Audoin-Rouzeau, Combattre, Paris, Seuil, 2008 et Lawrence Keeley, War before Civilisation. The Myth of Peaceful Savage, Oxford University Press, 1996. 
militaire ${ }^{20}$. Celle-ci avait su contenir le pouvoir d'effroi des innombrables carnages perpétrés à travers l'Europe les décennies précédentes. Parce que fortement édulcorée sous les célébrations du sacrifice héroïque, la guerre était restée pourvoyeuse des plus hautes valeurs morales et esthétiques. Or, c'est précisément cette sacralisation de l'expérience combattante et cette force de déréalisation qui caractérisent au mieux l'ère du «mythe de guerre » qui, selon George Mosse, s'est ouverte avec les guerres de la Révolution et a continué de s'épanouir jusqu'à la Grande Guerre au moins ${ }^{21}$. Avec l'armée révolutionnaire, la figure du citoyen-soldat cessa de véhiculer l'image inquiétante de la soldatesque de jadis. Autrefois jugé indigne - en dehors des grades d'officiers réservés à la seule noblesse -, le métier des armes avait soudain acquis une respectabilité nouvelle. Les buts de guerre concernaient désormais tout un chacun et non les seuls monarques. Plus qu'aucune autre, la figure de l'engagé volontaire est devenue l'objet d'un culte au long cours : celui du héros ayant choisi la guerre et ses sacrifices plutôt que de les subir. Le soldat mort au champ d'honneur avait fait son entrée au panthéon de la nation et réveillé l'antique imaginaire de la «belle mort ". L'émergence d'une abondante littérature de témoignages guerriers signale aussi les liens profonds qui se nouaient alors entre l'expérience de guerre et le moi romantique $^{22}$. Tels étaient les caractéristiques majeures de cette mystique guerrière ${ }^{23}$, qui, poussée à l'extrême, donna parfois à ces expériences un caractère de fêtte ${ }^{24}$, tant pouvait être grande l'excitation ressentie à l'approche des théâtres d'opérations.

Un élément plus tardif vint s'ajouter à cette concrétion mythique pour ne plus la quitter au cours du siècle : la guerre comme voyage, comme exploration de contrées lointaines et pittoresques, comme rencontre de populations mystérieuses, déroutantes et fascinantes à la fois. George Mosse eut l'intuition que l'aventure de Byron et des philhellènes dans la guerre gréco-turque, de par le légendaire qu'elle suscita, marqua, plus que les expéditions napoléoniennes en Égypte et en Russie, l'émergence d'une relation inédite à la guerre et au voyage ${ }^{25}$. Notre travail a creusé et confirmé cette hypothèse d'une "butte-témoin ${ }^{26}$ ". Un tel propos, bien sûr, ne vise pas à dire que la tentation des guerres exotiques ait été absolument nouvelle. Le temps des croisés comme celui des conquistadors véhiculèrent d'innombrables figures de guerriersvoyageurs qui restèrent autant de modèles pour les jeunes en mal d'évasion. Mais au cours des années 1820, la guerre et le voyage combinèrent leurs attraits d'une façon

20. Luigi Mascili Migliorini, Le Mythe du héros. France et Italie après la chute de Napoléon, Paris, Nouveau Monde éditions, 2002.

21. George L. Mosse, Fallen soldiers : Reshaping the Memory of the World's Wars, New York, Oxford University Press, 1990.

22. Charles Taylor, Sources of the Self. The Making of Modern Identity, Cambridge, Harvard University Press, 1989.

23. David Bell, ouvr. cité. ; Jean-Paul Bertaud, La Révolution armée. Les Soldats-citoyens et la Révolution française, Robert Laffont, 1979 ; Alan Forrest, Napoleon's Men. The Soldiers of the Revolution and Empire, Londres/New York, Hambledon, 2002 ; John A. Lynn, The Bayonnets of the Republic, Chicago, Westview Press, 1984.

24. Sur les rapports entre la guerre et la fête : Roger Caillois, Bellone ou la Pente de la guerre (1963), Paris, Flammarion, 2012, p. 235-244.

25. George Mosse, Fallen soldiers, ouvr. cité, p. 31.

26. Hervé Mazurel, Vertiges de la guerre. Byron, les philhellènes et le mirage grec, Paris, Les Belles Lettres, 2013. 
neuve et que les guerres napoléoniennes n'avaient qu'esquissé2 ${ }^{27}$. D'abord parce que l'imaginaire et l'exercice de la guerre en sont sortis profondément transformés, ensuite parce que le voyage, l'exotisme et bientôt l'aventure prirent des formes radicalement neuves ${ }^{28}$. Avec Byron s'épanouit une attirance pour les espaces moins connus, plus sauvages, plus dangereux aussi. Aux codes du voyage classique succédèrent ceux du voyage romantique, centrés sur l'écoute de soi au contact des populations autochtones et des paysages grandioses. Naissaient ainsi de nouvelles modalités d'appréciation des espaces naturels. Jadis, le désert brûlant, la haute mer, la forêt profonde ou encore les montagnes escarpées étaient perçus comme des espaces laids et hostiles, profondément répulsifs. Mais l'esthétique du sublime, le goût des paysages de chaos, la recherche de la vibration du moi à leur contact en firent, à l'époque romantique, des espaces désirés, intimement recherchés ${ }^{29}$. Cette profonde révolution de la culture du voyage, en rencontrant celle qui avait affecté peu auparavant l'esthétique de la guerre, fit ainsi surgir dans la palette historique des rêveries et des sentiments une tentation nouvelle, toute d'exaltation guerrière et de rêverie géographique, qui travailla en profondeur l'histoire occidentale au temps des conquêtes coloniales : celle de l'aventure guerrière lointaine, poursuivie au-delà des mers, jusque dans les contrées les plus inhospitalières et reculées du monde.

Ce désir inédit s'exprima en fait tout au long du siècle, dans ce slogan décliné à foison sur les affiches de recrutement : "Engagez-vous, vous verrez du pays! »affiches sur fond desquelles sont représentés ici de jeunes marins accostant des îles à la végétation luxuriante et aux populations demi-nues, là des fantassins se promenant, soleil couchant, dans les rues d'une paisible Médina. Sans rendre le service populaire, ce procédé attira du moins un nombre important de volontaires, au sein des élites comme des milieux populaires. Sa constante réitération, entre les années 1890 et les années 1930, dit bien d'ailleurs la persévérance de cet imaginaire. La curiosité des Français, jeunes et vieux, ne cessait de croître pour ces histoires d'aventuriers militaires s'enfonçant, tel Francis Garnier sur le Mékong, dans l'épaisse jungle indochinoise, explorant, tels Charles de Foucauld et Ernest Psichari, les immensités de pierre et de sable du désert saharien, fendant encore à pied, des semaines durant, comme le commandant Marchand, la moiteur de la forêt africaine ${ }^{30}$. La savane, le désert, les jungles, les plaines vertigineuses et les fleuves noirs - tous ces décors naturels signifiant l'Ailleurs - exerçaient un puissant envoûtement sur des lecteurs que fascinaient ces pénibles progressions à travers ces territoires hostiles, mais pleins de promesses. De

27. Nathalie Petiteau, «Les voyages des hommes de la Grande Armée : de la vie militaire aux pratiques de la mobilité géographique », dans Nicolas Bourguinat, Sylvain Venayre (dir.), Voyager en Europe, de Humbolt à Stendhal, Paris, Nouveau Monde, 2007, p. 345-363.

28. «Le Siècle du voyage », Sociétés et représentations, ${ }^{\circ}$ 21, 2006.

29. Alain Corbin, L'Homme dans le paysage, Paris, Textuel, 2001, p. 86.

30. Pierre Brullé, Judith Brouste, L’Appel du Sahara, Paris, Ed. Place des Victoires, 2011 ; Marc Michel, La mission Marchand 1895-1899, Paris, Mouton, 1972 ; Milton Osborne, River Road to China. The Mekong River Expedition, 1866-1873, Londres, Georges Allen, 1975. 
semblables projections assuraient à ces esprits aventureux une notoriété hautement recherchée d'eux. Tous savaient d'ailleurs trouver à leur retour un large auditoire ${ }^{31}$.

En somme, l'époque romantique, par son esthétisation de l'expérience combattante et son renouvellement des figures du voyage, conjuguait différemment le goût de la guerre et la tentation du voyage, jusqu'à donner naissance à un "dispositif affectif ${ }^{32}$ " de longue durée, qui vint soutenir le processus de conquête de la fin du siècle. Ralliant peu à peu le peuple français à l'idée impériale ${ }^{33}$, il multiplia ici et là les «vocations coloniales $^{34} "$.

\section{L'APPEL DES CONQUÊTES COLONIALES}

Tous les hommes exerçant outre-mer ne partirent pas de leur plein gré. Sur place, certains souffraient d'un sentiment d'exil, voire mouraient de "nostalgie ${ }^{35}$ ". Mais, parmi les officiers, beaucoup furent ceux qu'attirèrent outre-mer l'esprit de découverte et la course aux aventures. La majorité partit avec l'espoir d'y faire la totalité de leur carrière ; d'autres, nombreux également, firent à leur retour le siège des bureaux ministériels dans l'espoir d'une réaffectation. Et si ce désir des guerres lointaines circula prioritairement au sein des élites militaires, nourries de récits de voyage et de mémoires combattants, il joua également chez les simples soldats, parmi les humbles qu'attiraient au loin l'excitation de quitter un quotidien oppressant et la possibilité de voir du pays sans frais, sinon d'exprimer un certain goût des armes et de leur métier ${ }^{36}$.

À observer par exemple la composition de l'Armée d'Afrique ${ }^{37}$ ou celle des troupes de marines, en Afrique noire et à Madagascar ${ }^{38}$, il ressort que ces corps étaient composés de quasi-professionnels, d'hommes éprouvés, aguerris au métier de soldat ${ }^{39}$. Souvent originaires des zones tardivement intégrées à la France - le pays basque, la Corse, les zones côtières de l'Ouest et les provinces de l'Est -, les soldats, les volontaires en particulier, émanaient souvent des couches sociales les moins favorisées. S'ils étaient très majoritairement d'origine paysanne jusqu'au deux tiers du siècle, la part s'accrût ensuite des soldats originaires des villes et faubourgs. Les attiraient d'abord aux colonies l'assurance d'un toit, d'un couvert et d'une solde faible, mais

31. Edward Berenson, Les Héros de l'Empire. Brazza, Marchand, Lyautey, Gordon et Stanley à la conquête de l'Afrique (2011), Paris, Perrin, 2012.

32. Alain Corbin, «Intervention au colloque de New York », dans « Alain Corbin and the Writing of History », French Politics, Culture \& Society, vol. 22, n 2, 2004.

33. À suivre Edward Berenson, c'est la fascination de ces héros de l'Empire qui permit de populariser un certain idéal colonial auprès de masses initialement peu concernées. Edward Berenson, ouvr. cité, p. 33.

34. Jacques Frémeaux, De quoi fut fait l'Empire. Les guerres coloniales au XIX $X^{e}$ siècle, Paris, CNRS éditions, 2010, p. 96.

35. Thomas Dodman, « Mourir de nostalgie en Algérie française, 1830-1880 », Annales HSS, 2011/3, p. 743-784.

36. Jacques Frémeaux, ouvr. cité, p. 96.

37. Nom donné à l'ensemble des troupes stationnées en Afrique du Nord.

38. Les deux fusionnent en 1900 sous la dénomination de « troupes coloniales », désormais rattachées au ministère de la Guerre et non plus de la Marine. On parlera à l'avenir de « la Coloniale ».

39. Lorsqu'en métropole s'institue le service universel en 1905 , on convient que les conscrits ne pourront être envoyés aux colonies. 
régulière. Beaucoup étaient avides d'action, supportant mal l'ennui et la discipline de la caserne ${ }^{40}$. Des perspectives de carrière existaient pour ceux que le sérieux promettait aux grades de sous-officiers. Nul doute également que beaucoup appréciaient de jouir d'une domination qui leur était refusée en métropole, soit celle de l'encadrement des troupes « indigènes » qui peuplaient « la Coloniale », soit simplement d'incarner le conquérant face à des populations asservies et apeurées. Si l'image des soldats coloniaux demeurait peu flatteuse en métropole (on les décrivait en proie à toutes les licences - jeu, alcool et prostituées), le topos d'un recrutement parmi les seuls « mauvais garçons" des bas-fonds est assurément excessif ${ }^{41}$.

Partir outre-mer était par ailleurs l'assurance d'une vie plus ouverte, déchargée des contraintes sociales et familiales, et de voir ses revenus arrondis par des indemnités diverses, sinon par des pillages. Tout cela jouait pour les hommes comme pour les officiers. Apparentées aux anciennes prises de mer, les parts enlevées à l'ennemi étaient des sources de profits non négligeables. L'essentiel allait au Trésor public et aux besoins de l'armée, mais les sommes restantes, distribuées selon les grades, constituaient des avantages financiers bien réels. Lors de la prise de la Smala d'Abd El Kader au printemps 1843, la part de butin d'un simple spahi s'élevait à 1500 francs $^{42}$. Lors de l'expédition de Formose de 1884, les marins ramenèrent dans leurs cales des objets d'extrême valeur : bronzes, ivoires, faïences, porcelaines ${ }^{43} \ldots$ Les fortunes amassées par les officiers furent plus considérables encore. On connaît le scandale suscité par les pillages du Palais d'été à Pékin à l'automne 1860 par le corps expéditionnaire franco-britannique ${ }^{44}$, lequel valut au général Antoine Cousin-Montauban, devenu comte de Palikao, une réputation de pilleur invétéré, déjà gagnée d'ailleurs en Algérie, tout comme Achille de Saint-Arnaud accusé lui aussi de s'y être considérablement enrichi.

En plus du désir "de faire colonne» et de voir du pays, l'appel de la fortune joua donc son rôle. Mais cet intéressement matériel était vu comme un juste retour des choses, tant la vie aux colonies était rude et pleine de dangers. Chacun, en effet, se trouvait dans une situation d'extrême vulnérabilité face aux maladies tropicales à la malaria notamment. L'Afrique de l'Ouest fut ainsi surnommée « la tombe de l'homme blanc ». Et si les progrès de l'hygiène, la quinine et une plus grande attention aux zones d'intervention firent diminuer la mortalité dans le dernier tiers du siècle, les maladies demeurèrent la cause principale de décès jusqu'à la campagne du Maroc qui vit la majorité des morts l'être par suite d'actes guerriers ${ }^{45}$. De ces campagnes outre-mer restait l'image héroïsante de guerres contre la nature ${ }^{46}$.

40. Odile Roynette, "Bons pour le service ». L'expérience de la caserne à la fin du XIX e siècle, Paris, Belin, 2000.

41. Jacques Frémeaux, ouvr. cité, p. 114.

42. Ibid., p. 99.

43. Commandant Thirion, L'Expédition de Formose. Souvenir d'une Soldat, Paris, Lavauzelle, 1897, p. 23.

44. Bernard Brizay, Le Sac du palais d'été. Seconde guerre de l'Opium, Paris, Éditions du Rocher, 2003.

45. Henri Wesseling, Les Empires coloniaux européens 1815-1919, Paris, Gallimard, 2009, p. 81.

46. Douglas Porch, Wars of Empire, London, Cassel and company, 2000, p. 100. 


\section{LE MILITAIRE, LE HÉROS ET L'AVENTURIER}

Si les scandales liés aux pillages et aux exactions de la conquête - celui, par exemple, suscité par les massacres de la mission Afrique Centrale-Tchad, dite VouletChanoine ${ }^{47}$ - ternirent au tournant du siècle l'image de ces soldats des lointains, ils ne remirent pas foncièrement en cause l'héroïsation à l'œuvre.

C'est d'abord que l'on voulait voir dans ces pénétrations impériales, et cette domestication de l'inconnu, une parfaite œuvre mâle. Ces patriotes, bravant tant de périls, étaient offerts au public comme autant de modèles de virilité. L'éclat de la conquête d'un si vaste empire permettait aux Français de renouer avec l'épique napoléonien et d'apaiser l'humiliant souvenir de la défaite de 1870. En ces théâtres lointains, il était permis de reconstruire la fierté française. Le public, que fascinaient les atlas, y voyait également une échappatoire à l'atmosphère étouffante d'une France petite bourgeoise, matérialiste et casanière. Les militaires, eux, échappaient non seulement au morne enlisement des casernes, mais à cette armée moderne devenue une organisation bureaucratique, broyant les qualités de courage et d'audace. Tout au contraire, l'espace colonial leur offrait la liberté de mouvement, l'occasion de se distinguer, de faire valoir son esprit d'initiative et sa capacité au commandement. À la lenteur des avancements en métropole s'opposait la rapidité des promotions coloniales, comme le montrent les progressions d'un Gallieni au Soudan ou plus tard d'un Marchand. De plus, bien des officiers coloniaux, tels Lyautey, Mangin ou Archinard, bénéficièrent à leur retour de postes à hautes responsabilités.

Intéressant est ici le cas de Jean-Baptiste Marchand qui, après Fachoda et malgré l'humiliation subie face au rival anglais, fut accueilli par une foule en liesse à Paris, le 10 juin 1899. On se précipita gare de Lyon pour voir, toucher, parler à Marchand et à ses hommes. Avoir tenu tête à Kitchener avait presque transformé cette défaite en victoire. En ces heures d'intenses rivalités impériales, Marchand incarnait non seulement le parfait héros patriotique mais également l'idéal de ces officiers baroudeurs, explorateurs romantiques, défricheurs de terres inconnues, durs au mal mais pétris d'ardeur et d'idéal. L'incroyable progression de sa mission Congo-Nil avait marqué les esprits. Elle lui avait fait parcourir, en moins de deux ans et en dépit d'innombrables obstacles naturels, quatre mille cinq cents kilomètres à travers l'Afrique centrale. Aussi ce militaire avait-il réussi à réunir sur sa personne le prestige du héros et le charisme de l'aventurier qui, rappelons-le, n'avaient pas toujours été liés.

À l'époque romantique, en effet, la figure de l'aventurier n'était aucunement associée à ces images positives qui caractérisent le tournant du XX $\mathrm{X}^{\mathrm{e}}$ siècle $\mathrm{e}^{48}$. Au sortir des guerres de la Révolution et de l'Empire, elle était associée au mercenaire. L'aventurier désignait en réalité le contretype même du guerrier hérö̈que idéal, incarné par le soldat-citoyen et, plus encore, par l'engagé volontaire. Ce n'est qu'au fil du siècle grâce à Byron et à Garibaldi - que ces polarités commencèrent à s'inverser, rapprochant

47. Henri Wesseling, Le Partage de l'Afrique, Paris, Gallimard, 2002, p. 410-13.

48. Sylvain Venayre, La Gloire de l'aventure. Genèse d'une mystique moderne, 1850-1940, Paris, Aubier, 2002. 
les figures antagonistes du héros et de l'aventurier. Avant que ne s'impose « l'Aventure avec un grand $\mathrm{A}$ ", dégagée de toute finalité utilitaire ${ }^{49}$, ces militaires des lointains, convoitant d'un même élan les charmes de la guerre et du voyage d'exploration, incarnèrent l'aventure par excellence. Rimbaud lui-même y céda momentanément, en s'embarquant pour Java au dépôt d'enrôlement colonial hollandais d'Hardewijk "le caniveau de l'Europe »- avant de déserter dans d'obscures circonstances ${ }^{50}$. Offerts à l'admiration publique par la presse quotidienne, l'illustration et la littérature, ces militaires énergiques et ambitieux, ces hommes à la barbe de rigueur, au maintien grave et au détachement hautain, capables d'une vie jugée plus pleine, aux traits plus accusés, furent ainsi érigés en modèles d'exemplarité en ces temps de nationalisme exacerbé et, bientôt d'enthousiasme colonial.

Cette soudaine absence de guerres sur le continent européen à partir de 1815 ne fut-elle pas l'une des sources d'où jaillit la multiplication des conflits lointains orchestrés par les Français et les Européens au cours du XIX ${ }^{\mathrm{e}}$ siècle ? Car la disparition des champs de bataille en Europe entravait la reproduction du modèle militaro-viril issu des années 1792-1815 et avivait les nostalgies et les frustrations de ceux qui portaient justement «le mythe de guerre ». On connaît la somme des arguments mobilisés pour rendre raison des conquêtes impériales : la grandeur de la nation, la mission civilisatrice ou le « fardeau de l'homme blanc » cher à Kipling, la conquête de débouchés et de matières premières, voire le produit de raisons sociales (les colonies comme exutoire à la guerre civile). Toutes ces raisons sont capitales, leur hiérarchisation n'étant fonction que des choix historiographiques. Mais l'une nous paraît manquer à l'appel, qu'il faut chercher du côté de l'histoire des sensibilités et des imaginaires. Car cette grande ruée coloniale n'a été rendue possible que parce qu'elle s'est aussi appuyée sur cet idéal de virilité et ce goût de la guerre qui lui était consubstantiel, eux-mêmes mêlés à une forte soif de dépaysement et d'aventures. En somme, sans la montée au cours du siècle de ce désir de guerres lointaines, sans ces imaginations stimulées à l'extrême par ces récits épiques, par ces faits d'armes mythifiés par les publicistes et les romanciers, il est finalement douteux que l'Europe eût entrepris des conquêtes d'une telle ampleur.

(Université de Bourgogne)

49. Ibid.

50. Louis-Charles Damais, « Arthur Rimbaud à Java », Bulletin de la Société des Etudes Indochinoises, XLII, n 4 , Saigon, 1967, p. 335-339. 